\title{
La implementación de los sistemas de control de gestión en las empresas de nueva creación: Una revisión de la literatura
}

\section{Raúl González Castro}

Cámara de Comercio, Industria y Servicio de Ourense

El objetivo de este trabajo es una revisión literaria de la relación entre los sistemas de control de gestión y el porqué del uso de los mismos en las empresas de nueva creación. Con este fin, se han revisado 24 artículos publicados en 11 revistas en el período 1967-2015. Se definen los resultados en tres categorías: los motivos de la adopción, cuáles son y cómo actúan las variables de fomento de la adopción, y las consecuencias. Se analizan el estado actual de la investigación y las líneas de investigación futuras recogidas por la literatura recopilada.

Palabras clave: Sistemas de control de gestión, emprendimiento, revisión de la literatura, start-up

\section{The Implementation of Management Control Systems in Start-Up Companies: A Review of the Literature}

The objective of this work is a literature review of the relationship between management control systems and why the use of them in newly created companies. To this end, we have reviewed 24 articles published in 11 journals in the period 1967-2015. The results are defined in three categories: the reasons for adoption, they are and how they work variables promoting the adoption, and the consequences. The current status of research and future research collected by the literature collected is analyzed.

Keywords: Management control systems, entrepreneurship, literature review, start up.

\section{A implementaçáo de sistemas de controle de gestáo em empresas start-up: uma revisão da literatura}

O objetivo deste artigo é uma revisão da literatura sobre a relação entre os sistemas de controlo de gestâo e por que o uso delesem empresas recém-criadas. Para este fim, nós analisamos 24 artigos publicados em 11 revistas no período 19672015. os resultados são definidos emtrês categorias: as razôes para adoção, eles são e como eles funcionam variáveis que promovem a adoção e as consequências. o estado actual da investigação e futuras pesquisas coletadas pela literatura recolhida é analisada.

Palavras chave: Sistemas de controlo de gestão, empreendedorismo, revisão da literatura, o arranque 


\section{Introducción}

Las empresas de nueva fundación nacen de aquellas ideas empresariales que los emprendedores llevan al mercado y se convierten en fuente de la renovación del tejido empresarial, en la medida que aportan cambios en el mercado con nuevos productos o servicios, promueven cambios en la cadena de valor de mercado, o actúan sobre las escaseces del mismo. En general, ayudan a generar el progreso económico, social y tecnológico (Dávila y Oyon, 2009; Drucker 1985; Nevin y otros, 2011). En todo el proceso de creación de la empresa, los emprendedores deben tomar decisiones con un alto nivel de incertidumbre (Shane y Venkataraman, 2000). En este marco, se encuentran los start-up, un tipo de empresas innovadoras caracterizadas por su potencial de rápido crecimiento, desarrollo de productos sofisticados, actividad centrada en mercados altamente competitivos e inestables, y generalmente con acceso a financiación de capital riesgo (Dávila, Foster y Jia, 2014; Granlund y Taipaleenmäki 2005). Es un perfil que supera la mera creación de empresas, y no está solo marcada por años desde la fundación o por un determinado tamaño.

En la actualidad, no existe un consenso unánime en la literatura sobre el papel que desempeñan los sistemas de control gestión en este tipo de empresas. Además, no se han realizado estudios integrativos que permitan entender mejor esta cuestión. El propósito de este trabajo es proporcionar un marco en torno a las variables que explican la adopción, la intensidad y las consecuencias de la presencia de los sistemas de control de gestión (SCG) en empresas de nueva fundación, incluidos los start-up. Para ello, se realiza una revisión sistemática e integrativa de la literatura con análisis de conclusiones. La búsqueda en la literatura identifica un total de 24 artículos de 11 revistas diferentes desde 1967 hasta 2015.
Este estudio contribuye a la literatura sometiendo a un examen analítico los trabajos existentes que vinculan el sistema de control de gestión con la actividad de empresas de nueva creación y start-up. Además, se sintetizan las variables que relacionan la presencia y la intensidad de uso de los SCG, y las consecuencias de su utilización. La organización del artículo, tras la introducción, tiene una segunda sección, en la que se presentan las definiciones de SCG. En la tercera sección, explicamos cómo hemos diseñado y realizado la búsqueda. En la cuarta, presentamos una descripción de los contenidos encontrados. En la quinta sección, analizamos la literatura, $y$, en la sexta, sistematizamos los aportes y las oportunidades de investigación futuras. Por último, en la séptima sección, definimos las limitaciones de la revisión.

\section{Definición de sistemas de control de gestión}

Para este trabajo, se asume la formulación de Simons (1995), que define los sistemas de control de gestión como «las rutinas formales, basadas en la información y la gestión de procedimientos de utilidad para mantener o alterar patrones en las actividades de la organización». Malmi y Brown (2008) señalan que los SCG son «completos sistemas, normas, prácticas, valores y otras actividades de gestión que se ponen en marcha con el fin de dirigir el comportamiento del empleado». Por su parte, Dávila, Foster y Jia (2014) definen SCG como sistemas formalizados de protocolos institucionalizados y rutinas diseńadas para la motivación, el monitoreo; estos también abarcan los mecanismos de medida de los comportamientos de los gerentes y empleados, así como la asistencia al control y gestión de la información para la toma de decisiones.

En el nacimiento del SCG, este era considerado como una herramienta para desarrollar los objetivos nacidos 
en la planificación estratégica (Anthony, 1965). Con Gavetti y Levinthal (2000), se trasladan los SCG a la etapa de ejecución y se convierten en herramientas de corrección de desviaciones. Gordon y Narayanan (1984) reconocen la necesidad de las empresas de afrontar el control en presencia de un contexto de incertidumbre, propio de las empresas motivo del trabajo. En respuesta, se ha conceptualizado el sistema de control interactivo (Abernethy y Brownell, 1999). En este contexto, se genera el debate científico que se afronta en este trabajo: el papel que asumen los SCG en la gestión de las empresas de nueva creación. Por un lado, el cuerpo científico tradicional se sitúa en contradicción con la dinámica propia de las empresas de nueva fundación. En esta versión, los SCG son limitadores de éxitos y crecimiento de las nuevas empresas (Quinn, 1980). Por otro lado, se encuentran nuevas tendencias que defienden los SCG como herramientas de control adecuadas para la gestión de la inestabilidad (eso sí, exigiendo la evolución de nuevas herramientas) frente a la visión tradicional del uso de los SCG, como medio de control de gestión de forma jerarquizada e inflexible que puede limitar los procesos creativos propios de la innovación (Amabile, 1998).

Esta nueva visión utiliza otro perfil de herramientas de SCG, que las considera dinámicas, como el caso de Ouchi (1979) con el control del clan, basado en normas sociales en los departamentos de I + D. Abernethy y Lillis (1995) encuentran que las relaciones personales de «contacto espontáneo»y «enlace integrador» permiten dominar el proceso de control. Por tanto, los sistemas de control en presencia de incertidumbre se desagregan en herramientas de corte cultural (Tushman y O’Reilly, 1996), la dinámica de comunicación (Allen, 1970) o el liderazgo (Clark y Fujimoto, 1991). Con Malmi y Brown (2008), surge la alternativa de entender los controles como un «sistema» en que los componentes pueden ser diseńados y aplicados por diferentes individuos, y no tienen por qué estar en coordinación (Songini, Gnan y Malmi, 2013; Malmi y Brown, 2008).

Existen múltiples clasificaciones de las distintas herramientas de los SCG. Para nuestro trabajo, elegiremos las estructuras denominadas palancas de control de Simons (1995), debido a que es la clasificación más utilizada en los distintos estudios de la muestra, al analizar la tensión que existe en la gestión entre sistemas de creación y sistemas de medición. Simons (1995) identifica cuatro palancas de control de SCG formales: sistemas de creencias, sistemas de control de diagnóstico (SCD), sistemas de control interactivos (SCI) y sistemas de límites. Estos medios de control pueden ser usados por los administradores para la gestión del control y la innovación.

El sistema de creencias se considera como un sistema formal que habilita a los directivos para definir, comunicar y reforzar los valores básicos, el propósito, los objetivos y el destino de la empresa (Simons, 1995). Los sistemas de creencias son el medio para establecer límites y reglas explícitos en las organizaciones, que deben ser respetados por todos los miembros de la organización (Simons, 1995). Generalmente, se reflejan mediante normas, códigos de conductas o directrices de trabajo.

Los sistemas de control de diagnóstico son el medio de retroalimentación formal de los distintos sistemas utilizados para evaluar y hacer seguimiento de los objetivos de la organización (Simmons, 1991). Los mecanismos definidos incluyen los controles contables internos, planes de negocio, o presupuesto (Simons, 1995, 1994, 1991, 1987a, 1987b).

Los sistemas de control interactivos son sistemas formales utilizados para la toma de decisiones por parte de la alta dirección (Simons, 1995). Cualquier sistema 
de control de diagnóstico se convierte en interactivo al centrar su enfoque en los intereses de la dirección y en el fomento de la acción de aprendizaje de la organización (Simons, 1995). Simons determina que el trabajo simultáneo de SCD y SCI genera un proceso de «tensión dinámica» que, en situación, de equilibrio es capaz de apoyar la consecución de la estrategia definida y a la vez permite identificar las acciones necesarias para definir nuevas estrategias eficientes (Simons, 1995), así como ayuda a la gestión de la incertidumbre (Simons, 1995).

Los sistemas de límite determinan lo aceptable, y la estrategia de participación de los miembros en la organización y sus límites. Se establece mediante creencias, procedimientos o normas formales. Es un sistema burocrático, a partir del cual se establecen normas comunes con resultados duales, coactivas por superación de los límites, pero también de guía útil para la gestión.

\section{Diseño de la búsqueda}

En esta sección, se presentan los procedimientos metodológicos utilizados en la construcción y el análisis de la cartera bibliográfica seleccionada. Se ha desarrollado una investigación de carácter teórico, clasificada como estudio bibliográfico o revisión sistemática (Denyer y Tranfield, 2009), que analiza los estudios ya publicados sobre el tema (Creswell, 2009). Los objetivos son exploratorios y descriptivos, mediante la búsqueda de información específica sobre lo que se está estudiando.

El método utilizado para llevar a cabo la investigación bibliográfica fue compuesto por tres etapas diferentes: la recopilación de datos, el análisis de datos y la síntesis de los resultados (Rousseau, Manning y Denyer ,2008; Tranfield, Denyer y Smart, 2003). Siguiendo el trabajo de Lueg y Radlach (2016), en la etapa de

recopilación, se han seguido una serie de pasos para identificar los estudios buscados en el área de trabajo. El primer paso ha sido la definición del criterio de búsqueda, para lo cual se ha determinado que los estudios deben tratar el fenómeno de la relación entre SCG en nuevas empresas y en start-up. Se han realizado varias búsquedas en la base de datos ScienceDirect, que es una base de datos de resúmenes y citas de fuentes científicas bibliográficas y de información en Internet a nivel académico. ScienceDirect contiene más de 12 millones de artículos de consulta.

Debido a la diferente terminología de SCG acerca de nuevas empresas y de start-up, se han realizado varias cadenas de búsqueda, unido al uso del asterisco como elemento comodín dentro del título y el resumen. Los términos utilizados se basan en la siguiente consulta: («mcs» and «entrepreneurship») o («entrepreneurship» and "management accounting systems»), o ("start up» and "management accounting systems») o («start up» and "MCS») y («System Control» and «start up» o "Controller» and «Start up»). En el punto de partida, hemos buscado en las 25 revistas utilizadas por Hoque (2014) por su vínculo en el mundo de la contabilidad y los sistemas de gestión cerrando el período de búsqueda en el año 2015. Tras una primera búsqueda y tras la lectura somera de título, abstract y palabras claves, hemos realizado una selección de 66 artículos de 22 revistas.

El segundo paso ha sido una revisión completa de los artículos seleccionados, en la que se ha comprobado su referencia clara de uso de SCG en sistemas de empresas de nueva creación/Start-up. La selección queda reducida a un total de 19 artículos y 7 revistas. Posteriormente, en una tercera etapa, se aplicó el «enfoque de ascendencia» (Cooper, 1982). Esto permitió la inclusión de nuevos artículos en idioma inglés de interés para el trabajo. Hemos seguido las 
referencias seleccionadas de estudios empíricos listados por Science Direct. Sobre esa base, se incluyeron los artículos que cumplían los criterios de inclusión, en concreto, cinco artículos nuevos y cuatro revistas añadidas. De este modo, hemos terminado la búsqueda con 24 artículos y 11 revistas del período 1967 a 2015 (Apéndice 1). Se ha realizado un mapa de los artículos de forma intuitiva identificando las variables que incitan a su adopción y grado de intensidad, sobre la base de la clasificación de Malmi y Brown (2008). Esta labor nos permite aportar a la investigación científica una visión global del estado actual del cuerpo académico, en la que se identifica en el conjunto de la investigación, las variables que incitan a la adopción de los SCG en las nuevas empresas/start up, sus efectos ante los distintos sistemas de control y el grado de intensidad de los mismos.

\section{Descripción del análisis}

Analizamos las frecuencias y las características en los 24 estudios, a partir de lo cual podemos ver la evolución de la relación en los años de trabajo de la investigación 1967-2015.

\section{Gráfico 1. Panel A}

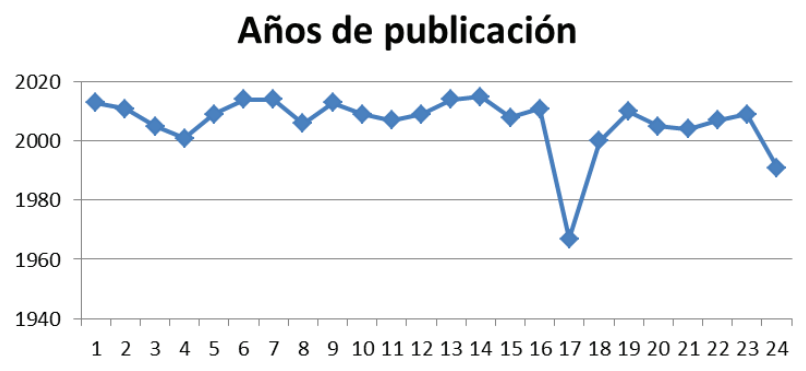

Fuente: Elaboración propia a partir de los datos del análisis.

Como se puede ver en el Panel A, el artículo más antiguo es del año 1967, pero el grueso de las publicaciones se ha realizado desde el cambio del siglo (2000),
22 publicaciones (91\%); en concreto, se muestra un proceso más agudo a partir del año 2010, con 9 publicaciones (37\%). En los años recientes, mayor número de estudios aceptan las ventajas de la presencia de los SCG en las empresas de nueva creación/start-up en los primeros años de la fundación.

\section{Gráfico 2. Panel B}

\section{Perfil de revistas}

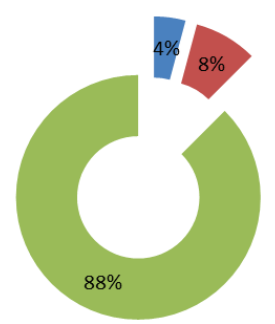

= sociales

" empresa

contabilidad

Fuente: Elaboración propia a partir de los datos del análisis.

Como se puede observar en el Panel B, el perfil de revistas seleccionado es principalmente de temática contable $(88 \%)$, debido a la temática y criterios de inclusión determinados. European Accounting Review es la revista con mayor número de artículos aportados. Solo el $4 \%$ proceden de revistas de temática social. El origen, principalmente, corresponde a EE.UU. (71\%) y el resto es de origen europeo (ver Panel C).

\section{Grráfico 3. Panel C}

\section{Origen de la publicación}

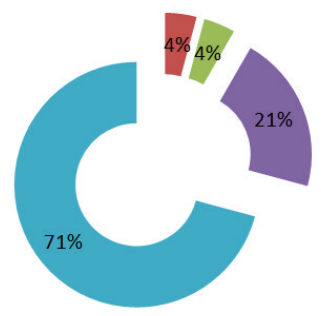

- EEUU

- Dinamarca

Multipaís

- Europa

EEUU 


\section{Contenido del análisis}

\subsection{Método}

El trabajo busca responder a las siguientes preguntas (i): ¿Cuáles son las consecuencias de la implantación de los SCG?, (ii) ¿cuáles son las variables de fomento de la adopción de los SCG en las empresas de nueva creación?, y (iii) ¿cuáles son las variables generadoras de intensidad en las relaciones de SCG en nuevas empresas?

Con los resultados de la evaluación, hemos desarrollado un marco conceptual que da respuesta a las preguntas, a partir de lo cual hemos determinado una serie de tipos de consecuencias. De esta manera, nos es posible analizar las consecuencias de capacidades organizacionales y las consecuencias de rendimiento económico. Algunos ejemplos de reacciones pueden ser motivación, cooperación o creatividad. En el caso de consecuencias de capacidades organizaciones, podemos hablar de procesos de gestión flexibles que permiten procesos de innovación; de rápido crecimiento; de aceptación del cambio; y, en general, el desarrollo de estrategias competitivas. En el caso de las consecuencias de rendimientos económicos, nos referimos a resultados financieros y no financieros, debido a que se consideran tanto rendimientos financieros como rendimientos organizacionales. Las variables analizadas proceden tanto de origen interno como externo.

\subsection{Teorías}

El mapa conceptual identifica tres teorías sobre las que se basa el trabajo científico: la teoría de la agencia, la teoría de contingencia y teoría del ciclo de vida. La teoría de agencia responde a dos fenómenos. En primer lugar, se ha utilizado para explicar cómo la reducción de las asimetrías de información dentro de los niveles de la organización puede mejorar el rendimiento organizacional (Stede, Chow y Lin, 2006; Eisenhardt, 1989; Jensen y Meckling, 1976; Jensen y Murphy, 1990). En segundo lugar, la teoría marca los criterios de necesidad de sistemas de medida y motivación individuales para mejorar el rendimiento organizacional global estableciendo metas e incentivos. Estas medidas atienden las necesidades de la información sobre expectativas de los inversores externos (Feltham y Xie, 1994).

\section{Gráfico 4. Mapa conceptual}

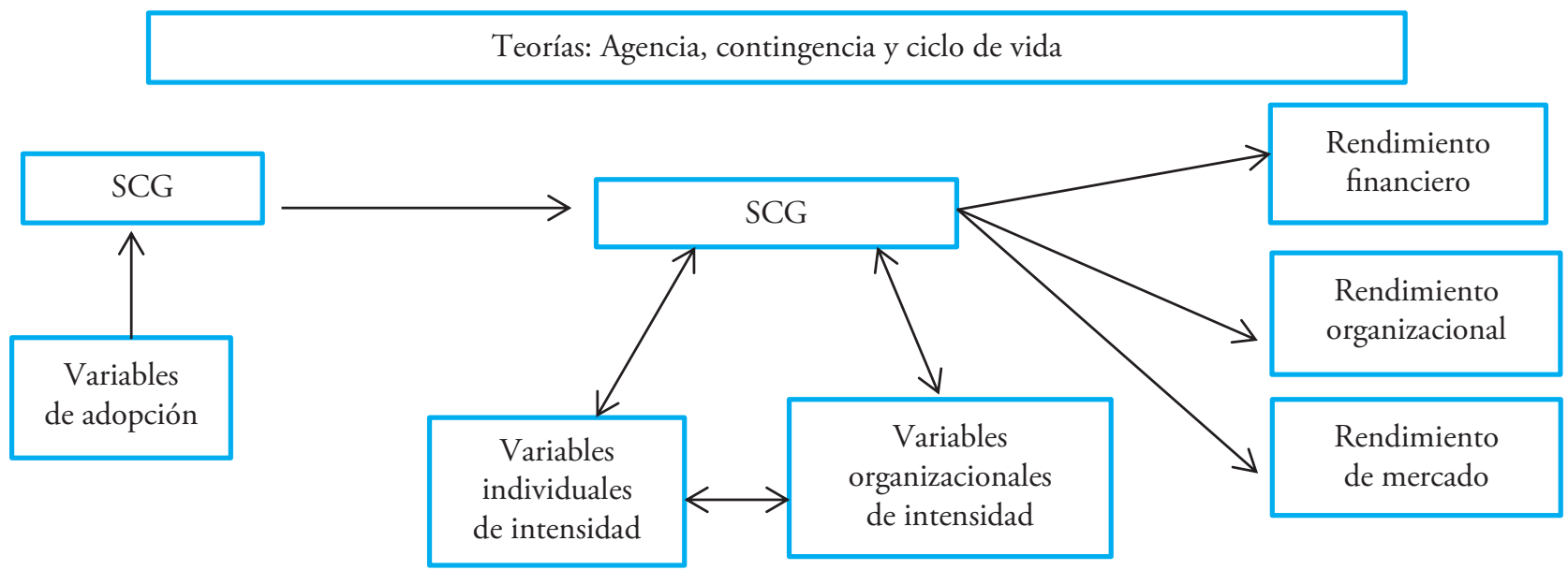


La teoría de la contingencia de las organizaciones predice que las características de una organización determinan la creación de sus sistemas de gestión y medición de resultados dependiendo de las contingencias específicas (Otley, 1980). Por tanto, la teoría de la contingencia determina que no hay mejor diseño de sistemas, sino que varía en función de las variables de la empresa (Sandino, 2007). El diseño del paquete de sistemas de control de gestión debe ser individual teniendo en cuenta variables como la estrategia o la presencia de la incertidumbre (Hoque, 2014). En relación con la teoría del ciclo de vida, Moore y Yuen (2011) reconocen que la configuración organizacional de las empresas tiene distintas dimensiones por sus características internas y externas, y provoca la evolución del estado de situación a lo largo del ciclo de vida. Los distintos modelos de la teoría del ciclo de vida aceptan el dinamismo de las empresas y lo explican como un fenómeno en evolución, condicionado por las estrategias, las estructuras, las áreas críticas, las problemáticas y las condiciones del medio. Como consecuencia, este modelo permite predecir los estados que las empresas van afrontar en el tiempo, y demuestra que las empresas son organizaciones que responden a los continuos cambios internos y externos.

\subsection{Variables de adopción}

Los trabajos seleccionados identifican que pueden existir causas internas y externas que fomentan la incorporación de SCG, como las necesidades de gestión de información para apoyar el control y la toma de decisiones, mostrar su solvencia ante inversores o su presentación como legitimadores ante terceras partes. La presencia de capital riesgo y la necesidad de gestionar la alineación de los objetivos de la empresa y de los empleados para alcanzar el éxito financiero se convierte en un porqué para la implementación de sistemas de control.

\subsubsection{Tamaño}

Greiner (1998) sugiere que la aparición de SCG es consecuencia de "una crisis de liderazgo", situación en la que el aumento de empleados exige la incorporación de sistemas de control. Esto supone pasar de sistemas informales a sistemas formales para reducir los costes de gestión de la dirección, y mejorar la interacción entre la dirección y un mayor número de empleados. En ese sentido, los sistemas formales son adoptados para mejorar la coordinación de las organizaciones y mejorar la gestión de dirección. La relación de tamaño y SCG es positiva, pero puede tener efectos diferentes en función del SCG elegido. El tamaño incentiva los procesos de cultura organizacional y sistemas formales de control de objetivos, $y$, en general, la presencia de sistemas de control formales de recompensa (Dávila, 2005, 2007; Moore y Yuen, 2001).

\subsubsection{Edad}

La edad está asociada con la acumulación de experiencia y de aprendizaje con el paso del tiempo. Es una variable que permite aprender incorporando sistemas de control que se van modificando en función del tipo de la experimentación de la organización (Dávila, 2005; Moore y Yuen, 2001). Edad y tamaño, cuando actúan de forma conjunta, aumentan el incentivo de la implantación de SCG.

\subsubsection{Reemplazo del fundador}

La tercera variable es el reemplazo del fundador por un nuevo CEO. Se identifica como una etapa clave para el paso del proceso informal de gestión a una etapa formal (Dávila, 2005). Es la fase en que se profesionaliza la gestión de la empresa con la entrada de nuevos empleados, y la aprobación de nuevos objetivos, que requieren la profesionalidad de la alta dirección y, por lo tanto, el fomento del control de gestión. 
En la medida que los «[f]undadores son probablemente inadecuados para ser gestores» (Greiner, 1998), los trabajos analizados reconocen el proceso de incorporación de un CEO y la sustitución del fundador como un paso hacia la profesionalización de la dirección, lo cual es una variable de adopción de SCG. Esto mejora el proceso de gestión y de aprendizaje (Dávila, 2005), unido a la presencia de personal con capacidad de conocimiento para gestionar dichos SCG.

\subsubsection{Capital riesgo}

El capital riesgo es considerado como fuente de financiación económica, pero también de conocimiento de gestión y de redes de contactos para la empresa. Al mismo tiempo, son exigentes en la calidad de la información de control de la misma con el fin de medir la rentabilidad de su inversión y un mayor control sobre la incertidumbre (Granlund y Taipaleenmäki, 2005; Dávila, 2005; Dávila y Foster, 2005, 2007). Son, por tanto, incentivadores de la adopción de SCG, puesto que las demandas específicas sobre gestión de la información que requieren los gestores de capital riesgo también pueden conducir a la incorporación de SCG. La experiencia de los gestores de SCG puede influir en la adopción de tales sistemas (Adler y Chen, 2011). Las cuatro anteriores variables (edad, tamaño, reemplazo del fundador y presencia de capital riesgo) son las que se muestran con mayor grado de repetición y de eficiencia como factores incentivadores de la presencia de SCG en la muestra de trabajos.

\subsubsection{Otras variables}

Además de las variables anteriores, debemos tener en cuenta otras que también inciden en la adopción de sistemas de control de gestión. Tal es el caso de la legitimidad, variable incentivadora externa vinculada con el reconocimiento de terceras partes, como ocurre con las inversiones, los clientes, los proveedores, entre otros (Dávila, 2005). Otra variable es la internacionalización. Dávila y Foster (2009) determinan que la internacionalización, con el efecto de aumento de unidades de negocio y tamaño de mercado, exige la presencia de SCG con el fin de poder atender, y coordinar la gestión y la información necesarias. Asimismo, la mayor parte de los estudios analizados vinculan los procesos de adopción con la evolución del ciclo de vida de las empresas y aceptan la tesis de que, a medida que cambian las etapas, también lo hacen las estrategias y las estructuras de las organizaciones (Chandler, 1962), asumiendo las etapas del modelo de Miller y Friesen $(1984)^{1}$.

\subsection{Variables de intensidad}

La configuración organizacional se refiere a una dimensión multidimensional de distintas conceptualizaciones, marcada por distintos factores externos (tecnologías o entorno) e internos (estrategia, cultura, estructura, o procesos) (Moores y Yuan, 2001).

\subsubsection{Estrategia}

Los trabajos aceptan que la presencia de sistemas de control de gestión mejora los debates directivos sobre las líneas estratégicas a definir y ayuda a focalizar las actividades de la dirección en los procesos clave de las organizaciones (Jazayeri y Scapens, 2008). El hallazgo acepta la relevancia de la relación entre estrategia y presencia de SCG, en especial, en la gestión de los recursos humanos y, en menor medida, en la relación con los inversores (Dávila, 2005).

1 Esto se observa en Greiner (1998); Moore y Yuen (2001); Saeb y Smith (2014); Dávila, Foster y Oyon (2009); Cassar (2009); Granlund y Taipaleenmäki (2005); Dunk (2004); Smith y Cordina (2014) (ver Apéndice 1). 


\subsubsection{Interorganización}

La investigación también identifica la variable interorganización como factor de mejora de los efectos de la presencia de SCG ya no solo dentro de la organización, sino también en colaboración con otras empresas, proveedoras o clientes. El trabajo de Wouters y Kirchberger (2015) analiza las relaciones de SCG más allá de la propia organización y encuentra evidencias de que los SCG son relevantes para generar procesos de gestión compartidos entre distintas organizaciones, lo que provoca un mayor valor añadido para el cliente final.

\subsubsection{Coordinación/Participación}

Se identifica la presencia de los SCG como un efecto positivo en el control y en la coordinación de los distintos niveles, tanto unidades de negocio como directivos, como unidades en distintas sedes o países, o incluso a nivel individual. Se encuentran evidencias de que los SCG permiten la coordinación y la socialización de los individuos, con lo cual facilitan la relación entre los distintos mandos de la organización. Asimismo, ayudan a asegurar la disponibilidad de la información pertinente para la mejora de la toma de decisiones de cara a la rentabilidad de negocio, así como también en la gestión de recursos humanos. La equidad en la gestión de personal aliena los objetivos individuales y del total de la organización, a partir de lo cual genera mejoras en los procesos de aprendizaje conjuntos y la resolución de conflictos en equipo (Dávila, 2005).

\subsubsection{Innovación}

Se revela que los SCG son incentivadores de la innovación al facilitar la adopción al cambio de forma natural y no impuesta por poder de la dirección. Los SCG fomentan la creación de una sistemática de creencias, cultura organizacional, y aceptación y gestión de la incertidumbre, que fomentan los resultados de cambio innovador.

\subsubsection{Motivación}

La investigación sobre el efecto motivacional de los sistemas de SCG ha obtenido resultados positivos sobre el rendimiento. Los estudios de Adler y Chen (2011) revelan que los sistemas de control de gestión mejoran la motivación de las personas hacia el logro de los objetivos estratégicos. En particular, encuentran que el grado de motivación de los empleados está influenciado por el grado de participación en el proceso de medición y su transparencia. A su vez, muestran que las características de los SCG pueden ser incentivadores de la creatividad generando distintos niveles simultáneos e intrínsecos de motivación. Eso sí, dependen del conjunto de SCG elegidos para el desarrollo. El trabajo considera que sistemas con alto grado de formalismos limitan la creatividad, a pesar del efecto motivación. Por su parte, sistemas de control burocráticos muestran una relación positiva entre el fomento de la motivación y la creatividad. Es decir, se evidencia una relación positiva en los casos de sistemas que ofertan información eficaz, y una medición y sistema de recompensas equitativo, lo cual está asociado a la libre determinación del individuo en sus actividades de creatividad.

\subsubsection{Toma de decisiones}

El impacto de SCG en la toma de decisiones de los directivos también ha sido objeto de atención en la literatura. La evidencia de la investigación sugiere que los administradores de SCG ayudan a crear mecanismos de gestión que permiten apropiarse de la información eficiente y que da lugar a la mejora del rendimiento. La eficiencia de los sistemas depende de las condiciones cognitivas de los gerentes, de los SCG adoptados y de las condiciones internas de las empresas (Dávila y Oyon, 2007). 


\subsubsection{Liderazgo}

Existe una relación bidireccional en el caso de liderazgo y SCG. Los SCG pueden modelar los estilos de liderazgo, pero al mismo tiempo las características de liderazgo del CEO o fundador son claves para la implantación de los SCG y su adecuada gestión.

\subsubsection{Cultura organizacional}

Se identifica que la implantación exitosa de sistemas de control de gestión está interrelacionada con el sistema de cultura organizacional presente y sus cambios futuros. La adecuación de la cultura presente y de los SCG implementados promueve los valores, y las creencias del fomento de la creatividad y la innovación para la mejora de los resultados. En ese sentido, los SCG son poderosos motores del cambio dentro de las empresas que incluso pueden modificar la cultura organizacional, pero, al mismo tiempo, la presencia de la cultura organizativa modera la evolución de los SCG (Henri, 2006, Dávila, 2005; Dávila, Foster y Jia, 2014).

\subsection{Consecuencias de capacidades de organización}

La literatura ha investigado el impacto de los SCG en las capacidades de organización en términos de estrategia, comunicación, capacidad estratégica, de gestión práctica y el control corporativo; cada una de estas consecuencias es revisada a continuación.

\subsubsection{Mejora de la estrategia}

La revisión y la implementación de los SCG encuentran efectos positivos en la estrategia organizacional. Los investigadores encuentran los SCG como herramientas eficaces para la definición de las estrategias y en los momentos de modificación de los mismos. Estos facilitan la implantación de la estrategia y su entendimiento entre los individuos que la forman. Además, los
SCG ayudan a marcar los pasos necesarios para alcanzar los objetivos estratégicos. En esa medida, los SCG influyen positivamente en la estrategia, pero el nivel de generación de efectos está sujeto a las características específicas del propio sistema adoptado (Dávila, 2005; Granlund y Taipaleenmaki, 2005; Auzair y LangfieldSmith, 2005; Dávila y Foster, 2007).

\subsubsection{Capacidades estratégicas: Innovación}

Algunos investigadores han determinado directamente el impacto de los SCG en una variedad de capacidades de la organización; la mayoría de ellos adoptan las palancas de Simons (1995). Dichos investigadores afirman que los SCG influyen en las capacidades estrategias (aprendizaje, creatividad o ventajas competitivas como la innovación) al influir en los procesos necesarios para su alcance. Con respecto al impacto de los SCG ante la innovación, los estudios determinan que los SCG son elementos válidos para la gestión del control del riesgo en los procesos de innovación, además de que pueden ser factores que moderen la implantación de sistema de cultura organizacional que faciliten la toma de decisiones en contextos de incertidumbre. La implementación de SCG interactivos puede favorecer la creatividad y la aparición de nuevas ideas de trabajo, así como ser una guía útil de los pasos a dar, facilitadores de singulares y múltiples formas internas de motivación hacia la mejora innovadora (Dávila, 2005; Adler y Chen, 2011; Dávila y Foster, 2009; Dávila, Foster y Oyon, 2009; Dávila y Oyon, 2009).

\subsubsection{Capacidades estratégicas: Aprendizaje}

Los SCG son necesarios al ser la herramienta que fomenta el aprendizaje. Dávila y Foster (2009) afirman que la dirección de las empresas acepta la implementación de SCG como factores de fomento de la innovación, y de los procesos hacia el cambio y 
el avance de la organización. Cabe anotar que el uso interactivo de los SCG promueve la capacidad de la organización no solo en términos de innovación y aprendizaje organizacional, sino también en la capacidad y orientación al mercado. Ahora, el uso dinámico (control) del SCG puede ser un limitador de tales ventajas; por lo tanto, se recomienda un uso equilibrado de ambos modelos. La tensión entre ambos usos de los SCG puede inclinar la balanza en las organizaciones en el uso de unas capacidades estratégicas sobre otras.

\subsubsection{Comunicación y gestión de la información}

Existe una asociación significativa en el impacto de los SCG en los procesos de gestión de la información, y de gestión de la comunicación interna y externa de la empresa. Estas relaciones positivas tienen un efecto final en la generación del rendimiento organizacional. Los trabajos de Dávila (2005), Dávila y Foster (2007), y Cassar (2009) ponen en valor los efectos beneficiosos de la gestión de la información y de los procesos de comunicación de los distintos ámbitos de la empresa. En particular, los SCG incentivan los trabajos en equipo y ayudan a la resolución de problemas de forma común. Por tanto, los SCG son valores claves para una buena disponibilidad de información para la toma de decisiones y funcionan como buenas correas de transmisión de la comunicación, siempre y cuando sean sistemas vivos y de comunicación en varios sentidos, que permitan la comunicación adecuada y no funjan exclusivamente como trasmisores de órdenes concretas.

\subsubsection{Gestión y control}

La literatura también explora las consecuencias de los SCG en las prácticas de gestión y control. El uso de la investigación sugiere que los sistemas de SCG son una eficaz herramienta en el control de los procesos de trabajo y de gestión de la comunicación de las tomas de decisión. A medida que se incorporan al «paquete» de SCG los procesos que forman la gestión y el control de las empresas, estos adquieren relevancia. Aun así, debemos tener en cuenta que procesos de control solo sancionadores no favorecen la expansión de SCG ni su eficiencia. Estos sistemas de control también están marcados por la experiencia previa de la dirección, la presencia de personal con conocimiento para su gestión, la capacidad de participación del personal y su implicación, la madurez del sistema, la cultura de la organización y la formación general del personal. De esta manera, el control y la gestión están moderados por una amplia diversidad de variables que determinan la naturaleza positiva o negativa de los SCG con las decisiones de control y de ejecución de la gestión. Además, la presencia de la incertidumbre puede ser un factor negativo en el caso de la presencia de sistemas de control de gestión con más peso formal o dinámico, y puede mostrar una relación positiva en el caso de SCG interactivos por su mejora en la transmisión de la información y la mejora de las relaciones interpersonales que permiten mayores sinergias para afrontar contextos inestables (Dávila, 2005; Eldredge, Iwaarden, Kearney, Wiele y Williams, 2013; Adler y Chen, 2011; Moore y Yuen, 2001; Toms, 2005; Sievers, Mokwa y Keienburg, 2013; Cassar y Ittner, 2009; Dávila, Foster y Jia, 2014; Ballou y Heitger, 2008; Baloff y Kennelly, 1967; Auzair y LangfieldSmith, 2005; Cassar, 2009; Fleischman y Carroll, 1991; Greiner, 1998; Cardinal, Sitkin y Long, 2004).

\subsection{Consecuencias para el rendimiento}

\subsubsection{Rendimientos financieros y no financieros}

Los sistemas de control de gestión influyen en todos los niveles de la organización y sus rendimientos. Tanto en los rendimientos financieros - por ejemplo, en rendimientos contables o en las mejoras 
en el mercado-, así como en los rendimientos no financieros — como puede ser la mejora de la percepción del cliente sobre la empresa-. Los estudios de Adler y Chen (2011), y Dávila y Foster (2009) muestran la mejora del rendimiento financiero. Por su parte, Auzair y Langfield-Smith (2005) evidencian la mejora de la relación percibida entre clientes y organización. Aun así, este campo de trabajo requiere un mayor grado de investigación.

\subsubsection{Los beneficios para la interrelación de empresas}

Solo un trabajo afronta este análisis: Wouters y Kirchberger (2015). De acuerdo con este, la presencia de los SCG permite la creación de una sistematización de trabajo que favorece la interorganización. Este proceso genera una serie de sinergias que influye positivamente en la aparición de rendimientos no financieros que favorecen al cliente final, lo cual mejora su percepción de la empresa.

\subsubsection{Los beneficios para el equipo de trabajo}

Dávila (2005), y Dávila, Foster y Jia (2014) afirman que los equipos de innovación mejoran en presencia de SCG, siempre condicionados por el modelo del "paquete» diseñado y desarrollado en un contexto en que la incertidumbre modera la mejoría.

\section{Contribuciones y líneas para investigaciones futuras}

En primer lugar, se utilizan criterios reproducibles para identificar los 24 estudios identificados en el ámbito de aplicación de los SCG y la iniciativa empresarial, y, luego, se estructura un mapa completo mediante la comparación entre sí y el análisis de las conclusiones. La investigación en este campo podrá beneficiarse de las categorizaciones, conclusiones, así como de las líneas futuras y lagunas de investigación identificadas. La corriente investigadora que considera los SCG como un factor de utilidad para la gestión de la incertidumbre, el desarrollo, y el crecimiento de las empresas de nueva creación o para los start-up se muestran en un proceso todavía incipiente. De esta manera, podemos poner en relevancia áreas que pueden ser de investigación futura, como es el caso del grado de las dimensiones que se deben considerar en la formación de un SCG adecuado para los objetivos de tales empresas. En muchas ocasiones, la investigación deja de lado las condiciones culturales que marcan la forma de actual del fundador o creador, o la influencia cultural del país ante el fomento de la iniciativa empresarial y la aceptación del fracaso (Auzair y Langfield-Smith, 2005). En general, el cuerpo de investigación pone de relieve una falta de amplitud en el análisis de los factores internos y externos que impulsan la adopción de los mecanismos de control en las empresas de nueva iniciativa. Además, se identifican carencias de sofisticación en los estudios analizados; por ejemplo, es evidente la necesidad de identificar cuál debe ser la secuencia de adopción de los mecanismos de control, el grado de intensidad en el uso de cada uno, cuáles son las fórmulas para equilibrar en las empresas la relación entre los sistemas formales y los sistemas informales, o cuáles son las contingencias que definen el estilo de los SGC implantados (Auzair y Langfield-Smith, 2005; Dávila y Foster, 2007). Debemos encaminar la investigación a un mayor nivel de profundidad, tanto ampliando el tiempo de observación, puesto que se reconoce que los SCG implantados aumentan su complejidad con el tiempo (Dávila y Foster, 2009), así como analizando la actuación de los individuos y su comportamiento frente a los sistemas de control elegidos, es decir, el papel de los recursos humanos como gestores e implementadores de SCG. Es de interés el estudio de la 
influencia que terceras partes asesoras pueden asumir en las herramientas de SGC implantados. Por otro lado, existen carencias en el conocimiento de las configuraciones óptimas de SCG que permitan un mejor rendimiento económico. A su vez, se detectan limitaciones metodológicas; por ejemplo, los trabajos reconocen que es necesario ampliar la muestra de los encuestados con mayor cantidad de países, y con un mayor nivel de sectores y gamas de empresas, así como con presencia de fuentes de financiación tradicionales. También, se requiere un mayor nivel de propiedades psicométricas con el fin de poder mejorar los análisis y testar las hipótesis hasta ahora concluidas (Dunk, 2004; Dávila, 2005; Dávila y Foster, 2009).

\section{Limitaciones del trabajo}

El enfoque de revisión de la literatura utilizada en este artículo no está exento de limitaciones; existe el riesgo de omitir estudios pertinentes o de introducir errores por motivos de interpretación personal de la distinción de las clasificaciones del tipo de SCG (Lueg y Radlach, 2016). Incluso, puede producir más generalidades (Rousseau y otros, 2008).

La dificultad de encontrar una definición de empresa de nueva creación o de start-up de consenso entre los investigadores analizados complica las tareas de comparación y definición de conclusiones. Además, en los trabajos, se reconoce la falta de profundidad en la línea de investigación de las etapas del ciclo de vida y la falta de claridad en la distinción en el paso de una a otra, lo que dificulta el conocimiento sobre cuáles son los procesos de adopción de los SCG y el liderazgo de cada uno en cada etapa; esto obstaculiza un análisis homogéneo en la comparación de trabajos. Frente a ello, es necesario realizar una investigación más profunda y oportuna para el buen fin de la implementación de los SCG. Los trabajos analizados muestran la importancia potencial de las características individuales en el éxito de la actividad empresarial, pero estas no son abarcadas con suficiente sofisticación para obtener resultados definitivos ni concluyentes. Se reconoce la falta de conocimiento científico suficiente. En general, los estudios marcan historias positivas en la investigación entre la implantación de SCG y la iniciativa empresarial.

\section{Referencias}

Abernethy, Margaret A. y Anne Lillis (1995). The Impact of Manufacturing Flexibility on Management Control System Design. Accounting, Organizations and Society, 20(4), 241-258. https://doi.org/10.1016/0361-3682 (94)E0014-L

Abernethy, Margaret. A. y Peter Brownell (1999). The Role of Budgets in Organizations Facing Strategic Change: An Exploratory Study. Accounting, Organizations and Society, 24(39), 189-204.

Adler, Paul S. y Clara Xiaoling Chen (2011). Combining Creativity and Control: Understanding Individual Motivation in Large-Scale Collaborative Creativity. Accounting, Organizations and Society, 36, 63-85. https://doi.org/10.1016/j.aos.2011.02.002

Allen, Terry (1970). Communication Networks in R\&D Laboratories. R\&D Management, 1(1). https://doi. org/10.1111/j.1467-9310.1970.tb01193.x

Amabile, Teresa (1998). How to Kill Creativity. Harvard Business Review, 76(5), 76-87.

Anthony, Robert (1965). Planning and Control Systems: A Framework for Analysis. Boston, MA: Harvard Business School Press.

Auzair, Sofiah Md. y Kim Langfield-Smith (2005). The Effect of Service Process Type, Business Strategy and Life Cycle Stage on Bureaucratic MCS in Service Organizations. Management Accounting Research, 16, 399-421. https://doi.org/10.1016/j.mar.2005.04.003 
Baloff, Nicholas y John W. Kennelly (1967). Kofenya: The Role of Accounting Information in Managing the Risks of a New Business Journal of Accounting Research, Autumn, 131-143.

Ballou, Brian y Dan L. Heitger (2008). Kofenya: The Role of Accounting Information in Managing the Risks of a New Business. Issues in Accounting Education, 23(2), 211-228.

Cardinal, L. B., S. B. Sitkin y C. P. Long (2004). Balancing and Rebalancing in the Creation and Evolution of Organizational Control. Organization Science, 15(4), 411-431.

Cassar, Gavin (2009). Financial Statement and Projection Preparation in Start-Up Ventures. The Accounting Review, 84(1), 27-51. https://doi.org/10.2308/accr. 2009.84.1.27

Chandler, Alfred D. (1962). Strategy and Structure: Chapters in the History of the American Enterprise. Cambridge: Massachusetts Institute of Technology.

Clark, Kim B. y Takahiro Fujimoto (1991). Product Development Performance: Strategy, Organization, and Management in the World Auto Industry. Boston, MA: Harvard Business School Press.

Cooper, Harris M. (1982). Scientific Guidelines for Conducting Integrative Research Reviews. Review of Educational Research, 52(2), 291-302. https://doi. org/10.3102/00346543052002291

Creswell, Jhon (2009). Research Design: Qualitative, Quantitative and Mixed Methods Approaches. Tercera edición. Londres: Sage.

Dávila, Antonio (2005). An Exploratory Study on the Emergence of Management Control Systems: Formalizing Human Resources in Small Growing Firms. Accounting, Organizations and Society, 30, 223-248. https://doi.org/10.1016/j.aos.2004.05.006

Dávila, Antonio y Daniel Oyon (2007). Accounting, Innovation and Entrepreneurship. European $A c^{-}$ counting Review, 16(3), 665-666. https://doi.org/ 10.1080/09638180701507213
Dávila, Antonio y Daniel Oyon (2009). Introduction to the Special Section on Accounting, Innovation and Entrepreneurship. European Accounting Review, 18(2), 277-280. https://doi.org/10.1080/096381809 02924522

Dávila, Antonio y George Foster (2007). Management Control Systems in Early-Stage Startup Companies. The Accounting Review, 82(4), 907-937. https://doi. org/10.2308/accr.2007.82.4.907

Dávila, Antonio y George Foster (2009). Reasons for Management Control Systems Adoption: Insights from Product Development Systems Choice by Early-Stage Entrepreneurial Companies. Accounting, Organizations and Society, 34, 322-347. https://doi. org/10.1016/j.aos.2008.08.002

Dávila, Antonio, George Foster y Ning Jia (2014). The Valuation of Management Control Systems in StartUp Companies: International Field-Based Evidence. European Accounting Review, 24, 2015 - Issue 2, 207-239.

Dávila, Antonio, George Foster y Daniel Oyon (2009). Accounting and Control, Entrepreneurship and Innovation: Venturing into New Research Opportunities. European Accounting Review, 18(2), 281-311,

Denyer, David y David Tranfield (2009). Producing a Systematic Review. Cranfield: Organization Studies.

Drucker, Peter (1985). Innovation and Entrepreneurship: Practice and Principles. New York: Business \& Economics.

Dunk, Alan S. (2004). Product Life Cycle Cost Analysis: The Impact of Customer Profiling, Competitive Advantage, and Quality of IS Information. Management Accounting Research, 15, 401-414

Eisenhardt, Kathleen (1989). Building Theories from Case Study Research. Academy of Management Review, 14(4), 532-550.

Feltham, Georgia y Jianping Xie (1994). Performance Measure Congruity and Diversity in Multi-Task Principal/ Agent Relations. The Accounting Review, 69(3), 429-453.

Contabilidad y Negocios (12) 23, 2017 / ISSN 1992-1896 
Fleischman, Richard K. y John Carroll (1991). British Entrepreneurs and Pre-Industrial Revolution Evidence of Cost Management. The Accounting Review, (66, no. 2, pp. 361-375

Gavetti, Giovani y Daniel Levinthal (2000). Looking Forward and Looking Hackward: Cognitive and Experiential Search. Administrative Science Quartely, 45(1). https://doi.org/10.2307/2666981

Gordon, Lawrence y Vadake Narayanan (1984). Management Accounting Systems, Perceived Environmental Uncertainty and Organization Structure: An Empirical Investigation. Accounting, Organizations and Society, 9(1), 33-47. https://doi.org/10.1016/03613682(84)90028-X

Granlund, Markus y Jani TaipaleenmÁki (2005). Management Control and Controllership in New Economy Firms: A Life Cycle Perspective. Management Accounting Research, 16, 21-57. https://doi.org/10.1016/j. mar.2004.09.003

Greiner, Levis (1998). Evolution and Revolution as Organizations Grow. Harvard Business Review, 6, 55-64.

Henri, Jean-Francois (2006). Organizational Culture and Performance Measurement Systems. Accounting Organizations and Society, 31(1), 77-103. https:/doi. org/10.1016/j.aos.2004.10.003

Hoque, Zaquirul (2014). 20 Years of Studies on the Balanced Scorecard: Trends, Accomplishments, Gaps and Opportunities for Future Research. The British Accounting Review, 46. https://doi.org/10.1016/j. bar.2013.10.003

Jazayeri, Mehrdad y Robert Scapens (2008). The Business Values Scorecard within BAE Systems: The Evolution of a Performance Measurement System. The British Accounting Review, 40, 1. https://doi.org/10.1016/j. bar.2007.10.007

Jensen, M. C. y Kevin Murphy (1990). Performance Pay and Top-Management Incentives. Journal of Political Economy, 98(2), 225-264. https:/doi. org/10.1086/261677
Jensen,Michaely WillianMeckling(1976). TheoryoftheFirm: Managerial Behavior, Agency Costs and Ownership Structure. JournalofFinancialEconomics,3(4),305-360. https://doi.org/10.1016/0304-405X(76)90026-X

Lueg, Rainer y Ronny Radlach (2016). Managing Sustainable Development with Management Control Systems: A Literature Review. European Management Journal, 34(2), 158-171. https://doi.org/10.1016/j. emj.2015.11.005

Malmi, Teemu y David Brown (2008). Management Control Systems as a Package Opportunities, Challenges and Research Directions. Management Accounting Research, 19(4), 287-300. https://doi.org/10.1016/j. mar.2008.09.003

Miller, Danny y Peter. H. Friesen (1984). A Longitudinal Study of the Corporate Life Cycle. Management Science, 30(10), 1161-1183. https://doi.org/10.1287/ mnsc.30.10.1161

Moore, Ken y Susana Yuen (2001). Management Accounting System and Organizational Configuration: A Life-Cycle Perspective. Accounting Organizations and Society, 26, 351-389

Nevin, D., B. Ølknur Taútan y E. Öznur Gülen (2011). The Relationship between Entrepreneur's Level of Perceived Business-Related Fear and Business Performance. Procedia Social and Behavioral Sciences, 24.

Otley, David (1980). The Contingency Theory of Management Accounting: Achievement and Prognosis. Accounting, Organizations and Society, 5(4), 413-428. https://doi.org/10.1016/0361-3682(80)90040-9

Ouchi, William (1979). A Conceptual Framework for the Design of Organizational Control Mechanisms. Chapter. Readings in Accounting for Management Control. 63-82. https://doi.org/10.1007/978-1-4899-7138-8_4

Quinn, James Brian (1980). Managing Strategic Change. MIT Sloan. Management Review. Dartmouth, summer, July 15. 
Rousseau, Denise, Joshua Manning y David Denyer (2008). Evidence in Management and Organizational Science: Assembling the Field's Full Weight of Scientific Knowledge through Syntheses. Academy of Management Annals, 2(1). https://doi. org/10.1080/19416520802211651

Saeb, Nahs y Michael Smith (2014). Innovation in Entrepreneurial Organizations: A Platform for Contemporary Management Change and a Value Creator. The British Accounting Review, 46, 60-76.

Sandino, Tatiana (2007). Introducing the First Management Control Systems: Evidence from the Retail Sector. The Accounting Review, 82(1), 265-293. https:// doi.org/10.2308/accr.2007.82.1.265

Shane, Scott y Shane Venkataraman (2000). The Promise of Entrepreneurship as a Field of Research. Academy of Management Review, 25, 1045-1057. https://doi. org/10.5465/AMR.2000.2791611

Sievers, Soenke, Christopher F. Mokwa y George Keienburg (2013). The Relevance of Financial versus Non-Financial Information for the Valuation of Venture Capital-Backed Firms. European Accounting Review, Vol. 22, no. 3, pp. 467-511

Simons, Robert (1987). Accounting Control Systems and Business Strategy: An Empirical Analysis. Accounting, Organizations and Society, 12(4), 357-374. https:// doi.org/10.1016/0361-3682(87)90024-9
Songini, Lucrezia, Luca Gnan y Teemu Malmi (2013). The Role and Impact of Accounting in Family Business. Journal of Family Business Strategy, 4(2). https://doi. org/10.1016/j.jfbs.2013.04.002

Stede, Wim A. van der, Chen W. Chow y Tae W. Lin (2006). Strategy, Choice of Performance Measures, and Performance. Behavioral Research in Accounting, 18(1), 185-206. https://doi.org/10.2308/ bria.2006.18.1.185

Toms, Steven (2006). Accounting for Entrepreneurship: A Knowledge-Based View of the Firm. Critical Perspectives on Accounting, 17(2-3), 336-357.

Tranfield, David, David Denyer y Palminder Smart (2003). Towards a Methodology for Developing EvidenceInformed Management Knowledge by Means of Systematic Review. British Journal of Management, 14(3), 207-222. https://doi.org/10.1111/14678551.00375

Tushman, Michael y Charles O'Reilly (1996). The Ambidextrous Organizations: Managing Evolutionary and Revolutionary Change. California Management Review, 38(4), 8-30. https://doi.org/10.2307/41165852

Wouters, Marc y Markus A. Kirchberger (2015). Customer Value Propositions as Interorganizational Management Accounting to Support Customer Collaboration. Industrial Marketing Management, 46, 54-67. https:// doi.org/10.1016/j.indmarman.2015.01.005 


\section{Apéndice 1}

\begin{tabular}{|c|c|c|c|}
\hline Artículo & Año & Revista & Autor \\
\hline $\begin{array}{l}\text { Management Control Systems for Business Processes in Uncer- } \\
\text { tain Environments }\end{array}$ & 2013 & $\begin{array}{l}\text { International Journal of } \\
\text { Quality \& Reliability } \\
\text { Management, 31(1), 66-81 }\end{array}$ & $\begin{array}{l}\text { Stephen Eldridge, Jos van } \\
\text { Iwaarden, A.T. Kearney, } \\
\text { Ton van der Wiele y Roger } \\
\text { Williams }\end{array}$ \\
\hline $\begin{array}{l}\text { Combining Creativity and Control: Understanding Individual } \\
\text { Motivation in Large-Scale Collaborative Creativity }\end{array}$ & 2011 & $\begin{array}{l}\text { Accounting, Organizations and } \\
\text { Society, 36, 63-85 }\end{array}$ & $\begin{array}{l}\text { Paul S. Adler y Clara } \\
\text { Xiaoling Chen }\end{array}$ \\
\hline $\begin{array}{l}\text { An Exploratory Study on the Emergence of Management Control } \\
\text { Systems: Formalizing Human Resources in Small Growing Firms }\end{array}$ & 2005 & $\begin{array}{l}\text { Accounting, Organizations and } \\
\text { Society, 30, 223-248 }\end{array}$ & Antonio Dávila \\
\hline $\begin{array}{l}\text { Management Accounting System and Organizational Configura- } \\
\text { tion: A Life-Cycle Perspective }\end{array}$ & 2001 & $\begin{array}{l}\text { Accounting Organizations and } \\
\text { Society, 26, 351-389 }\end{array}$ & Ken Moore y Susana Yuen \\
\hline $\begin{array}{l}\text { Reasons for Management Control Systems Adoption: Insights } \\
\text { from Product Development Systems Choice by Early-Stage } \\
\text { Entrepreneurial Companies }\end{array}$ & 2009 & $\begin{array}{l}\text { Accounting, Organizations and } \\
\text { Society, 34, 322-347 }\end{array}$ & $\begin{array}{l}\text { Antonio Dávila y George } \\
\text { Foster }\end{array}$ \\
\hline $\begin{array}{l}\text { Accounting for Entrepreneurship: A Knowledge-Based View of } \\
\text { the Firm }\end{array}$ & 2006 & $\begin{array}{l}\text { Critical Perspectives on } \\
\text { Accounting }\end{array}$ & Steven Toms \\
\hline $\begin{array}{l}\text { The Relevance of Financial versus Non-Financial Information for } \\
\text { the Valuation of Venture Capital-Backed Firms }\end{array}$ & 2013 & $\begin{array}{l}\text { European Accounting Review, } \\
22(3), 467-511\end{array}$ & $\begin{array}{l}\text { Soenke Sievers, Christopher F. } \\
\text { Mokwa y George Keienburg }\end{array}$ \\
\hline $\begin{array}{l}\text { Accounting and Control, Entrepreneurship and Innovation: Ven- } \\
\text { turing into New Research Opportunities }\end{array}$ & 2009 & $\begin{array}{l}\text { European Accounting Review, } \\
18(2), 281-311\end{array}$ & $\begin{array}{l}\text { Antonio Dávila, George } \\
\text { Foster y Daniel Oyon }\end{array}$ \\
\hline $\begin{array}{l}\text { Introduction to the Special Section on Accounting, Innovation } \\
\text { and Entrepreneurship }\end{array}$ & 2009 & $\begin{array}{l}\text { European Accounting Review, } \\
18(2), 277-280\end{array}$ & Antonio Dávila y Daniel Oyon \\
\hline Accounting, Innovation and Entrepreneurship & 2007 & $\begin{array}{l}\text { European Accounting Review, } \\
16(3), 665-666\end{array}$ & Antonio Dávila y Daniel Oyon \\
\hline Initial Retention of External Accountants in Startup Ventures & 2009 & $\begin{array}{l}\text { European Accounting Review, } \\
18(2), 313-340\end{array}$ & $\begin{array}{l}\text { Gavin Cassar y } \\
\text { Christopher D. Ittner }\end{array}$ \\
\hline $\begin{array}{l}\text { The Valuation of Management Control Systems in } \\
\text { Start-Up Companies: International Field-Based Evidence }\end{array}$ & 2014 & $\begin{array}{l}\text { European Accounting Review } \\
\text { http://dx.doi.org/10.1080/09 } \\
638180.2014 .965720\end{array}$ & $\begin{array}{l}\text { Antonio Dávila, } \\
\text { George Foster y Ning Jia }\end{array}$ \\
\hline $\begin{array}{l}\text { Customer Value Propositions as Interorganizational Management } \\
\text { Accounting to Support Customer Collaboration }\end{array}$ & 2015 & $\begin{array}{l}\text { Industrial Marketing Manage- } \\
\text { ment, 46, 54-67 }\end{array}$ & $\begin{array}{l}\text { Marc Wouters y } \\
\text { Markus A. Kirchberger }\end{array}$ \\
\hline $\begin{array}{l}\text { Kofenya: The Role of Accounting Information in Managing the } \\
\text { Risks of a New Business }\end{array}$ & 2008 & $\begin{array}{l}\text { Issues in Accounting Education, } \\
23(2), 211-228\end{array}$ & $\begin{array}{l}\text { Brian Ballou y Dan L. } \\
\text { Heitger }\end{array}$ \\
\hline Accounting Implications of Product and Process Start-ups & 1967 & $\begin{array}{l}\text { Journal of Accounting Research, } \\
\text { Autumn, 131-143 }\end{array}$ & $\begin{array}{l}\text { Nicholas Baloff y John W. } \\
\text { Kennelly }\end{array}$ \\
\hline $\begin{array}{l}\text { Management Control and Controllership in New Economy firms. } \\
\text { A Life Cycle Perspective }\end{array}$ & 2005 & $\begin{array}{l}\text { Management Accounting } \\
\text { Research, 16, 21-57 }\end{array}$ & $\begin{array}{l}\text { Markus Granlund y } \\
\text { Jani Taipaleenmäki }\end{array}$ \\
\hline $\begin{array}{l}\text { The Effect of Service Process Type, Business Strategy and Life } \\
\text { Cycle Stage on Bureaucratic MCS in Service Organizations }\end{array}$ & 2005 & $\begin{array}{l}\text { Management Accounting } \\
\text { Research, 6, 399-421 }\end{array}$ & $\begin{array}{l}\text { Sofiah Md. Auzair y } \\
\text { Kim Langfield-Smith }\end{array}$ \\
\hline $\begin{array}{l}\text { Product Life Cycle Cost Analysis: The Impact of Customer Pro- } \\
\text { filing, Competitive Advantage, and Quality of IS Information }\end{array}$ & 2004 & $\begin{array}{l}\text { Management Accounting } \\
\text { Research, 15, 401-414 }\end{array}$ & Alan S. Dunk \\
\hline
\end{tabular}




\begin{tabular}{|l|l|l|l|}
\hline \multicolumn{1}{|c|}{ Artículo } & Año & \multicolumn{1}{|c|}{ Revista } & \multicolumn{1}{|c|}{ Autor } \\
\hline Management Control Systems in Early-Stage Startup Companies & 2007 & $\begin{array}{l}\text { The Accounting Review, 82(4), } \\
907-937\end{array}$ & $\begin{array}{l}\text { Antonio Dávila y George } \\
\text { Foster }\end{array}$ \\
\hline $\begin{array}{l}\text { Financial Statement and Projection Preparation in Start-Up } \\
\text { Ventures }\end{array}$ & 2009 & $\begin{array}{l}\text { The Accounting Review, 84(1), } \\
27-51\end{array}$ & Gavin Cassar \\
\hline $\begin{array}{l}\text { British Entrepreneurs and Pre-Industrial Revolution Evidence of } \\
\text { Cost Management }\end{array}$ & 1991 & $\begin{array}{l}\text { The Accounting Review, 66(2), } \\
361-375\end{array}$ & $\begin{array}{l}\text { Richard K. Fleischman y } \\
\text { John Carroll }\end{array}$ \\
\hline $\begin{array}{l}\text { Evolution and Revolution as Organizations Grow } \\
\text { Balancing and Rebalancing in the Creation and Evolution of }\end{array}$ & 2004 & $\begin{array}{l}\text { Harvard Business Review, 6, } \\
55-64 .\end{array}$ & L. E. Greiner \\
Organization Science, 15(4), & $\begin{array}{l}\text { L. B. Cardinal, S. B. Sitkin } \\
\text { y. P. Long }\end{array}$ \\
\hline
\end{tabular}

Fecha de recepción: 14 de junio de 2016 Fecha de aceptación: 07 de abril de 2017 Herero36@gmail.com 